\title{
AvaliaçäO dO ESTADO NUTRICIONAL E dO CONSUMO ALIMENTAR DE MULHERES NO CLIMATÉRIO
}

\author{
Regina das Neves Girão Montilla*, Maria de Fátima Nunes Marucci, José Mendes Aldrighi
}

Trabalho realizadono Centro de Saúde-Escola "Geraldo de Paula Souza" da Faculdade de Saúde Pública da Universidade de São Paulo

RESUMO - Objetivo. Avaliar o estado nutricional e o consumo alimentar de mulheres no climatério.

Métodos. Em um estudo transversal foram avaliadas 154 mulheres atendidas no Ambulatório de Saúde da Mulher Climatérica (ASMUC) do Centro de Saúde-Escola da Faculdade de Saúde Pública da Universidade de São Paulo. As variáveis analisadas foram Índice de Massa Corporal (IMC) e consumo alimentar (energia, distribuição percentual de proteínas, carboidratos e gorduras, vitaminas A e C, minerais cálcio e ferro). Para analisar valor energético da alimentação e minerais cálcio e ferro foram utilizados dois grupos etários (35-50 anos e 5I-65 anos). Para análise de IMC, vitaminas A e C e percentual de macronutrientes, foi considerada a população total estudada (35-65 anos). Em todas as variáveis foram utilizadas médias e desvios padrões.
Resultados. 0 IMC médio foi $29,3 \mathrm{~kg} / \mathrm{m}^{2}\left( \pm 6,2 \mathrm{~kg} / \mathrm{m}^{2}\right)$, com $75 \%$ da população acima do índice de normalidade. Quanto à energia, os resultados mostraram-se adequados para os dois grupos etários; quanto à distribuição percentual dos macronutrientes todos apresentaram-se inadequados; em relação às vitaminas e minerais, somente o ferro mostrou-se adequado.

Conclusão. A população avaliada apresenta riscos à saúde, face aos valores elevados de IMC e consumo inadequado de macronutrientes, cálcio, vitaminas $\mathrm{A}$ e $\mathrm{C}$.

UnITERMos: Climatério. Estado nutricional. Consumo alimentar. Índice de Massa Corporal (IMC). Obesidade. Osteoporose.

\section{INTRODUÇÃO}

Vários agravos à saúde constatados no climatério relacionam-se, na maioria das vezes, direta ou indiretamente com a ingestão inadequada de alimentos, quer seja em excesso ou deficiência por longos períodos; essa inadequação, por sua vez, constitui em importante fator de risco' para inúmeras doenças, tais como as cardiovasculares, a obesidade, a osteoporose, o câncer de cólon e de mama ${ }^{2,3,4}$.

No caso específico da mulher climatérica, os excessos nutricionais relacionam-se com o alto consumo de alimentos energéticos, principalmente provenientes de gorduras saturadas $^{2,3}$. Consoante Hill et al. ${ }^{5}$, a gordura proveniente da dieta é considerada determinante primária de obesidade, sendo esta mais prevalente em mulheres a partir do climatério ${ }^{6}$. Outros estudos revelam deficiência de nutrientes, como as vitaminas $A$ e $C^{4,7}$, o cál$\mathrm{cio}^{2,8-10}$ e o ferro ${ }^{4}$.

A existência de poucos estudos sobre nutrição em mulheres climatéricas reforça a ne-

\footnotetext{
*Correspondência:

Av. Coral, 280-Cond. Arujá 07400-000-Arujá-SP
}

cessidade de avançar nesta investigação. De fato, a própria Organização Mundial de Saúde $(\mathrm{OMS})^{11}$ recomenda pesquisas nessa área, a fim de melhor conhecer possíveis relações entre distúrbios alimentares e agravos à saúde. Como ocorre um aumento da prevalência de doenças crônicas no climatério, torna-se importante identificar a realidade nutricional da mulher nesse período, uma vez que a nutrição adequada é primordial para assegurar a saúde.

\section{Métodos}

Trata-se de estudo transversal, cuja população foi constituída por I 54 mulheres matriculadas no Ambulatório de Saúde da MuIher Climatérica (ASMUC) do Centro de Saúde-Escola "Geraldo de Paula Souza" da Faculdade de Saúde Pública da Universidade de São Paulo. Sendo o ASMUC um ambulatório de atendimento multiprofissional, todas as pacientes eram encaminhadas primeiramente à consulta médica, onde eram solicitados os exames necessários (dosagem hormonal, colesterol, triglicérides, glicemia, mamografia, densitometria óssea, etc). As mulheres entre 35 e 50 anos apresentavam ciclos menstruais num intervalo variável entre $24 \mathrm{e}$ 38 dias; o grupo após a menopausa, constituí- do de pacientes entre 51 e 65 anos, não menstruavam há pelo menos um ano e apresentavam níveis séricos de FSH superior a $30 \mathrm{mu} / \mathrm{ml}$. Após atendimento médico, as pacientes eram convidadas a participarem deste estudo e, em caso afirmativo, assinavam o termo de consentimento. As variáveis de estudo foram Índice de Massa Corporal e Consumo Alimentar (energia, distribuição de macronutrientes, cálcio, ferro, vitamina $\mathrm{Ae}$ C). Para energia, cálcio e ferro, a população foi distribuída em dois grupos (35-50 anos e 5 I-65 anos) com 77 mulheres em cada, tendo em vista que as recomendações dessas variáveis dietéticas são feitas conforme segmento etário. Para IMC, percentual de macronutrientes e vitaminas $\mathrm{Ae} C$, a análise foi feita considerando a população total, uma vez que não existe diferenciação nas recomendações dessas variáveis por grupo etário. A variável para analisar estado nutricional foi o Índice de Massa Corporal (IMC), calculado por meio do "software" "Virtual Nutri" desenvolvido por Philippi et al. ${ }^{12}$ a partir da relação peso $(\mathrm{kg}) /$ estatura $\left(\mathrm{m}^{2}\right)$. A avaliação da população por meio do IMC foi realizada, adotando-se o padrão de referência da OMS $1995^{13}$. Para verificação do consumo alimen- 
tar, utilizou-se o método de inquérito alimentar "recordatório de 24 horas", que consiste na quantificação das preparações e/ ou alimentos ingeridos durante o dia anterior a entrevista, baseando-se em medidas caseiras. Quanto ao consumo alimentar, as variáveis dietéticas foram energia (Valor Calórico Total - VCT), carboidratos, proteínas, gorduras, vitaminas $\mathrm{A}$ e $\mathrm{C}$, e os minerais cálcio e ferro. Os dados referentes ao consumo alimentar foram processados também pelo "software" "Virtual Nutri" 12 para obtenção do consumo médio de energia, de macronutrientes (proteínas, carboidratos e gordura) e micronutrientes (vitaminas e minerais), bem como da distribuição percentual dos macronutrientes e a adequação percentual das demais variáveis dietéticas (energia, vitaminas $\mathrm{A}$ e $\mathrm{C}$, cálcio e ferro). $\mathrm{O}$ padrão de referência utilizado para adequação energética foi o do National Resourch Council (NRC 1989)14, considerando adequada a dieta que apresentasse VCT entre $80 \%$ e $120 \%$ do recomendado. Para a distribuição percentual das proteínas, utilizouse a recomendação proposta por Young, Munro e Fukugawa, citados por Horwitz ${ }^{15}$, que consideram adequada a dieta que apresente percentuais entre $12 \%$ e I $4 \%$. Para as gorduras e os carboidratos, os parâmetros utilizados foram os valores sugeridos por Norum, Pederson e Wohl, também citados por Horwitz ${ }^{15}$, considerando adequados os seguintes percentuais: gorduras $-25 \%$ a $30 \%$; carboidratos - pelo menos $55 \%$ do VCT. Como os autores citados não mencionam o percentual máximo para carboidrato, ficou definido o valor de $63 \%$ como porcentagem máxima. A opção por este valor se deu em função da diferença entre $100 \%$ do VCT e as recomendações mínimas de proteínas e de gorduras. Quanto às vitaminas $\mathrm{Ae} C$ e o mineral ferro, foram utilizadas as recomendações do $\mathrm{NRC}^{14}$; em relação ao cálcio, utilizaram-se os valores sugeridos pela National Academy of Sciences $(\mathrm{NAS})^{10}$. A adequação das variáveis dietéticas (energia, vitaminas $\mathrm{A}$ e $\mathrm{C}$, cálcio e ferro) foi avaliada entre $80 \%$ e $120 \%$ das recomendações. Para todas as variáveis foram calculadas as respectivas médias e desvios padrões, utilizando o aplicativo Excel 2000 da Microsoft.

\begin{tabular}{|c|c|c|c|c|c|}
\hline \multicolumn{6}{|c|}{$\begin{array}{c}\text { Tabela I - Distribuição das mulheres com idade entre 35-65 anos, atendidas } \\
\text { em Centro de Saúde-Escola, segundo IMC, SP }\end{array}$} \\
\hline $\begin{array}{l}\mathrm{IMC} \\
\left(\mathrm{kg} / \mathrm{m}^{2}\right)\end{array}$ & $\begin{array}{l}\text { Classificação } \\
\text { segundo IMC }\end{array}$ & $\begin{array}{l}\text { Freqüência } \\
\text { absoluta }\end{array}$ & $\begin{array}{l}\text { Freqüência } \\
\text { relativa }\end{array}$ & $\begin{array}{l}\text { Média IMC } \\
\left(\mathrm{kg} / \mathrm{m}^{2}\right)\end{array}$ & $\mathrm{DP}\left(\mathrm{kg} / \mathrm{m}^{2}\right)$ \\
\hline $\begin{array}{l}\text { até } 18,4 \\
18,5-24,9 \\
25,0-29,9 \\
30,0-39,9 \\
\text { acima de } 40,0\end{array}$ & $\begin{array}{l}\text { MagrezaleveGrau I } \\
\text { Normal } \\
\text { Sobrepeso Grau I } \\
\text { Sobrepeso Grau } 2 \\
\text { Sobrepeso Grau3 }\end{array}$ & $\begin{array}{c}1 \\
37 \\
54 \\
53 \\
9\end{array}$ & $\begin{array}{c}1 \% \\
24 \% \\
35 \% \\
34 \% \\
6 \%\end{array}$ & $\begin{array}{l}18,2 \\
22,6 \\
27,7 \\
33,2 \\
45,0\end{array}$ & $\begin{array}{l}1,5 \\
1,5 \\
2,8 \\
3,5\end{array}$ \\
\hline TOTAL & & 154 & $100 \%$ & 29,3 & 6,2 \\
\hline
\end{tabular}

Tabela 2 - Percentual de adequação de consumo das variáveis dietéticas, desvio padrão e proporção de mulheres com consumo adequado, segundo variáveis dietéticas e grupo etário, SP

\begin{tabular}{cccc}
\hline $\begin{array}{c}\text { Variáveis } \\
\text { dietéticas }\end{array}$ & $\begin{array}{c}\text { Grupo etário } \\
\text { (anos) }\end{array}$ & $\begin{array}{c}\text { Percentual de } \\
\text { adequação e } \\
\text { Enesvio padrão(\%) }\end{array}$ & $\begin{array}{c}\text { Proporção de } \\
\text { mulheres com } \\
\text { adequação(\%) }\end{array}$ \\
Proténa & $35-50$ & $82,5( \pm 33,0)$ & 44,2 \\
Gordura & $51-65$ & $88,6( \pm 35,6)$ & 46,9 \\
Carboidrato & $35-65$ & $16,4( \pm 6,3)$ & 28 \\
Cálcio & $35-65$ & $51,4( \pm 12,2)$ & 15 \\
& $35-65$ & $32,2( \pm 10,3)$ & 17 \\
Ferro & $35-50$ & $64,0( \pm 64,0)$ & 14,3 \\
& $51-65$ & $51,0( \pm 62,2)$ & 14,5 \\
VitaminaA & $35-50$ & $108,1( \pm 108,8)$ & 31,5 \\
VitaminaC & $51-65$ & $113,0( \pm 59,0)$ & 21,7 \\
& $35-65$ & $120,7( \pm 99,0)$ & 18,2 \\
\hline
\end{tabular}

\section{Resultados}

Quanto ao IMC, 24\% da população apresentaram-se adequados em relação ao estado nutricional, tendo em vista que o IMC situou-se nos valores de normalidade; $35 \%$ foram classificados com sobrepeso graul,34\% com grau 2,6\% com grau 3 e $1 \%$ com magreza grau I. Portanto, $76 \%$ da população estudada apresentaram IMC inadequado, sendo $75 \%$ com sobrepeso e obesidade (Tabela I).

Quanto à energia, os percentuais médios foram adequados, ou seja, $82,5 \%( \pm 33,0 \%)$ e $88,6 \%( \pm 35,6 \%)$ para os grupos etários de 35-50 anos e 51-65 anos, respectivamente. Avaliando-se a proporção de mulheres com dieta adequada em energia, obteve-se $44,2 \%$ para o grupo etário dos $35-50$ anos e $46,9 \%$ para as mulheres acima de $5 \mathrm{I}$ anos (Tabela 2).

Os percentuais para os três macronutrientes (proteínas, carboidratos e gorduras) indicaram inadequação. Os nutrientes que ex- cederam aos percentuais recomendados foramas proteínas $(16,4 \% \pm 6,3 \%)$ eas gorduras (32,2\% $\pm 12,2 \%)$. Já os carboidratos apresentaram valores inferiores $(51,4 \% \pm 10,3 \%)$ à recomendação. Ao analisar a proporção da população com adequação nos macronutrientes, constata-se que somente $28 \%$ da população apresentaram quantidade adequada de proteína, $15 \%$ de gordura e $17 \%$ de carboidrato.

Quanto à adequação das vitaminas $\mathrm{Ae} \mathrm{C}$, observa-se na Tabela 2, que ambas atingiram valores médios superiores as recomendações. Em relação à proporção de mulheres com dieta adequada em vitamina $\mathrm{A} \mathrm{e} \mathrm{C}$, constataram-se $18,2 \%$ e $7,8 \%$, respectivamente.

Nos dados referentes ao cálcio dietético (640 mg e $610 \mathrm{mg}$ para os grupos dos 35-50 e $5 \mathrm{I}-65$ anos, respectivamente), os dois grupos etários apresentaram-se inadequados, sendo que o percentual de adequação para 0 
grupo de $35-50$ anos foi de $64 \%$ e para o outro grupo de $5 \mathrm{I}-65$ anos foi $51 \%$. Em relação à proporção da população com consumo adequado de cálcio dietético, os resultados foram $14,3 \%$ e $14,5 \%$ para os grupos etários dos 35-50 e 51-65 anos, respectivamente, revelando que a maior parte (mais de $85 \%$ em ambos os grupos) da população apresentou-se com consumo inadequado desse nutriente.

Quanto à adequação do ferro, verificase que em média o resultado apresentou-se adequado para os dois grupos etários (Tabela 2). Entretanto, apesar dessa adequação, somente $31,5 \%$ do grupo entre 35 50 anos e $21,7 \%$ para o grupo etário acima dos 50 estavam de acordo com a recomendação do NRC (1989) $)^{14}$.

\section{Discussão}

Os resultados sobre IMC mostraram sobrepeso em $75 \%$ da população avaliada; isso é extremamente preocupante, uma vez que o risco de morbi-mortalidade aumenta coma obesidade ${ }^{16,17}$. Esses resultados se assemelham com aqueles obtidos em outros estudos, tanto em países desenvolvidos como em desenvolvimento ${ }^{5,18-21}$.

Vários estudos revelam que mulheres a partir do início do climatério (40 anos) $)^{6,16,22}$ apresentam progressivo aumento de peso. De fato, isso foi comprovado pelo First National Health and Nutrition Examination Survey Follow-up Study ${ }^{23}$, que analisando 10.000 americanos, com idade entre 25-74 anos durante dez anos, constatou aumento de peso, tanto em homens como em mulheres.

Em relação à energia, esta apresentou valores de acordo com o estabelecido (80\%120\% das recomendações do NRC $1989^{14}$ ) para ambos os grupos etários; essa adequação, por sua vez, situou-se próxima ao limite inferior do estabelecido. Entretanto, como se obteve uma prevalência de $75 \%$ da população com IMC acima da normalidade $\left(29,3 \mathrm{~kg} / \mathrm{m}^{2}\right.$ $\left.\pm 6,2 \mathrm{~kg} / \mathrm{m}^{2}\right)$, ao se comparar o IMC com os valores energéticos da dieta, percebe-se que essas duas variáveis não apresentaram necessariamente a mesma tendência. Isso pode ser explicado por três razões: I) o IMC é um índice que avalia estado nutricional atual. Assim, ao analisar um indivíduo com IMC inadequado, significa que $\circ$ ganho ou a perda ponderal ocorreu ao longo do tempo, isto é, tanto sobrepeso, obesidade como magreza representam processos crônicos. Entretanto, a avaliação do inquérito alimentar pelo recordatório de 24 horas verificou quantitativamente a ingestão alimentar no dia anterior a entrevista, independente de seu estado nutricional atual, ou seja, é um dado recente e, portanto, não poderia exercer influência direta na obesidade; 2) como a partir da terceira década da vida ocorre diminuição do metabolismo basal, supõe-se que se esta redução não for acompanhada por queda no valor energético da dieta ou por elevação no dispêndio energético pela atividade física, ocorrerá um desequilibrio entre consumo e gasto, resultando em sobrepeso e obesidade a médio e longo prazo $0^{6,24}$. Frise-se que para a OMS" a atividade física éfundamental na prevençãoe controle da obesidade; 3) Como esse estudo foi realizado em um ambulatório multiprofissional, é possível que orientações nutricionais básicas dadas anteriormenteàs mulheres possam ter influenciado os resultados, pois muitas delas consumiam dietas hipocalóricas.

Quanto à distribuição dos macronutrientes, verifica-se que, apesar dos percentuais estarem bem próximos às recomendações, a maioria da população mostrou distribuição inadequada, principalmente quanto às gorduras e aos carboidratos. Esses resultados sugerem uma tendência pela preferência a alimentos gordurosos e protéicos. Apesar de não se tratar de questões relacionadas à mulher climatérica, Mondini \& Monteiro ${ }^{19}$ estudaram as modificações no padrão da alimentação urbana brasileira e perceberam uma tendência de aumento na participação relativa das gorduras e das proteínas. Essa preferência sabidamente é prejudicial à saúde da mulher climatérica, pois o excesso de gordura dietética favorece a obesidade, as doenças cardiovasculares, o câncer de mama e o de endométrio ${ }^{17,26,27}$, além do que o excesso protéico pode gerar aumento na excreção renal do cálcio, favorecendo a osteoporose ${ }^{2,28-32}$.

Hill et al. ${ }^{5}$ argumentam que a gordura dietética é determinante primária de obesidade. Entretanto, outros autores mostram que o percentual de energia proveniente das gorduras tem-se reduzido de forma expressiva nos últimos anos ${ }^{19,22}$, apesar do aumento na prevalência da obesidade ${ }^{33}$, sugerindo que a gordura seja somente um dos vários determinantes de uma "superalimentação".

Inúmeros estudos s $^{5,34,37}$ tentam explicar a relação entre o consumo exagerado de gordura dietética e a eclosão da obesidade; assim, dietas ricas em gorduras têm menor termogênese $\mathrm{e}^{35}$, maior eficiência no armazenamento ${ }^{5,36}$, menor poder de saciedade ${ }^{38,39}$ e são mais palatáveis do que alimentos de baixa densidade energética, ${ }^{5,39,40}$. Dessa forma, a redução na ingestão de gordura dietética parece ser efetiva na queda do peso corporal, mesmo sem restrição energética ${ }^{37}$. No entanto, Hill et al. ${ }^{5}$ mencionam que o maior efeito da dieta de baixo teor de gordura pode não ser a diminuição no ganho de peso, mas sim sua prevenção.

Quanto ao percentual de proteína dietética, verificou-se que a média esteve acima da recomendação; como o excesso protéico pode promover maior excreção renal de cálcio, esse fato torna-se particularmente importante na mulher após a menopausa, pois ela já apresenta progressiva perda de massa óssea ${ }^{28,29,31}$. Além do mais, outra complicação está no fato do consumo de cálcio revelar-se inadequado nos dois grupos etários (Tabela 2). Em um estudo recente ${ }^{41}$, verificou-se que a proteína poderia contribuir no ganho ósseo desde que o consumo de cálcio estivesse adequado. Para Heaney ${ }^{42}$, a proteína e o cálcio podem atuar no osso sinergicamente se ambos estiverem presentes na dieta em quantidades adequadas. Entretanto, a proteína pode tornar-se efetivamente antagonista para o metabolismo ósseo quando 0 consumo de cálcio é baixo.

A despeito do aumento na recomendação dietética do cálcio ${ }^{10,28}$, a média continua inadequada, comprometendo ainda mais a saúde óssea da mulher no climatério. Considera-se ainda que a proporção de mulheres com dieta adequada neste nutriente foi baixa em ambos os grupos etários ( $14,3 \%$ e $14,5 \%$ para os grupos 35-50 anos e 5I-65 anos, respectivamente). Esses resultados foram ainda inferiores do que aqueles obtidos por Galeazzi el a ${ }^{43}$, que verificou o consumo alimentar em cinco municípios brasileiros (Rio de Janeiro, Campinas, Curitiba, Goiânia e Ouro Preto). Neste estudo, o consumo médio de cálcio (em mulheres com idade de 45-65 anos) nas cinco cidades brasileiras foi de $917 \mathrm{mg}$ de cálcio e a proporção de mulheres com consumo de cál- 
cio adequado foi de $31,3 \%$. De acordo com Yates et a $\left.\right|^{10}$, o aumento na ingestão dietética deste mineral (1000-1200 mg), mesmo em mulheres pós-menopausais, poderia retardar a perda de cálcio ósseo.

Em relação ao ferro dietético, os resultados revelam que a média está adequada, com $90,5 \%( \pm 90,3)$ da recomendação para o grupo etário dos $35-50$ anos e I I2,8\% ( $\pm 58,8)$ para o grupo dos $51-65$ anos. Essa adequação decorre do consumo protéico elevado na dieta, uma vez que as principais fontes de ferro dietético advêm de certos alimentos protéicos (carnes em geral). De acordo com Felippe $\mathrm{Jr}^{44}$, é comum o consumo excessivo de ferro em adultos, idosos e em mulheres na menopausa. Entretanto, quando se avalia a proporção de mulheres (31,5\% para o grupo etário de $35-50$ anos e $21,7 \%$ para o grupo de $51-65$ anos) com dieta adequada em ferro e o desvio padrão, os resultados mostraram-se insatisfatórios, pois a maioria da população apresentou dieta inadequada em ferro.

$O$ percentual médio de vitamina A mostrou-se inadequado (I20,7\%), segundo os critérios adotados; entretanto, apesar desse resultado situar-se próximo à adequação máxima, somente $18,2 \%$ da população estava adequada em relação à vitamina $A$. Os demais $81,8 \%$ apresentaram inadequação, sendo que deste percentual, $47,4 \%$ estavam abaixo dos $80 \%$ da recomendação, ou seja, quase metade da população tinha consumo deficiente de vitamina A. É importante considerar que essa deficiência pode acarretar cegueira noturna, xeroftalmia, alterações cutâneas, e aumentar a susceptibilidade à carcinogênese ${ }^{7}$. Para os $34,4 \%$ (dos $81,8 \%$ ) da população que apresentaram consumo acima do recomendado, parece não ocorrer maior risco de agravos à saúde, pois não há na literatura casos conhecidos de toxicidade alimentar por essa vitamina ${ }^{7}$.

Quanto ao percentual de adequação da vitamina $\mathrm{C}$, o resultado de $201,5 \%$ sugere que a população consome dieta rica em ácido ascórbico, porém quando são avaliados o desvio padrão e a proporção da população com dieta adequada nesta vitamina, percebese nítida discrepância nos resultados. Assim, a parcela da população que estava com recomendação adequada em vitamina $C$ foi de somente $7,8 \%$. Os demais $92,2 \%$ com inadequação nessa vitamina foram divididos em $35,7 \%$ abaixo da recomendação mínima
(80\%) e $56,5 \%$ acima da recomendação máxima (I20\%). O consumo elevado de vitami$\mathrm{na} C$ dietética não promove qualquer problema, pois o excesso de ácido ascórbico dietético é excretado pelos rins ${ }^{7}$. Entretanto, estudos indicam que indivíduos que ingerem dietas com altos teores de ácido ascórbico (acima de $1000 \mathrm{mg} / \mathrm{dia}$ ), apresentam risco significativo de cálculos renais decorrentes de oxalato ${ }^{45}$. Esses resultados se assemelham aos de Galeazzi et al.$^{43}$ que confirmaram a mesma tendência de alto consumo de alimentos ricos em vitamina $C$, nos cinco municípios brasileiros pesquisados (Rio de Janeiro, Campinas, Ouro Preto, Goiânia e Curitiba).

\section{Conclusão}

Portanto, na avaliação do estado nutricional e do consumo alimentar, este estudo mostrou que as mulheres climatéricas alimentam-se de forma incorreta, principalmente do ponto de vista quantitativo. Vale ressaltar que o nutriente mais deficiente foi o cálcio, em especial para o grupo que necessita de maior atenção e por isso, programas de orientação nutricional devem fazer parte no atendimento à mulher climatérica. Além do mais, é fundamental que a educação nutricional deva ser estimulada na pré-escola, quando hábitos e atitudes estão em formação. Assim, uma alimentação adequada instituída precocemente e mantida continuamente representa um importante fator promotor de saúde e preventivo quanto aos agravos crônicos à saúde.

\section{SUMMARY}

\section{Nutritional status AND fOOd INTAKE ASSESSMENT OF CLIMACTERICS WOMEN}

BACKGROUND. To evaluate the nutritional status and food intake of climacterics women.

METHODS. In a transversal study were evaluated 154 women matriculated in the Clinic of the Climacteric Woman of the Health Center of Public Health College of the São Paulo University, Brazil. The analyzed variables were body mass index (BMI) and food intake (energy, percentual distribution of macronutrients, A and Cvitamins, calcium and iron minerals). To analyse food caloric value and calcium and iron minerals, were utilized two age groups (35-50 y and 5I-65 y). To analyse $B M I$, macronutrients percentage and $A$ and $C$ vitamins were utilized the total population (35-65 years of age). In all variables were calculated mean and standard deviation.

RESULTS. The mean BMI was $29.3 \mathrm{~kg} / \mathrm{m}^{2}$ $\left( \pm 6.2 \mathrm{~kg} / \mathrm{m}^{2}\right)$, with $75 \%$ of population above normality. About the energy, the results indicated adequacy to two age groups; about the distribution percentual of macronutrients, all were inadequate. In relation to the vitamins and minerals, all were inadequates, with exception of iron.

ConcLusION. The population studied presents risks to health owing to high value of $B M I$ and inadequate intake of macronutrients, calcium, $A$ and C vitamins. [Rev Assoc Med Bras 2003; 49(I): $91-5]$

KeYSWORDS: Climacterium. Nutritional status. Food intake. Body Mass Index (BMI). Obesity. Osteoporosis.

\section{REFERÊNCIAS}

I. Shuman JM. Nutrição no envelhecimento. In: Mahan LK, Krause ESS, editors. Alimentos, nutrição e dietoterapia. São Paulo: Roca; 1998. p. 294-3I5.

2. Feldman EB. Nutritional needs of climateric women. In: Notelovitz M, Van Keep P, editors. The climateric in perspective. London: MTP Press Limited; 1986. p. 27-40.

3. Sutnick M. Nutritional aspects of the menopause. In: Eskin BA, editor. The menopause: comprehensive management. 3th ed. New York: MacGraw Hill; 1994. p. I55-70.

4. Reimer A, Ellenbogen L, Feldman EB, Gallagher C, Rivlin R, Wagner P, et al. Nutrition. In: Notelovitz M, Vankeep P, editors. The climateric in perpective. London: MTP Press Limited; 1986. p. 353-60.

5. Hill JO, Melanson EL, Wyatt HT. Dietary fat intake and regulation of energy balance: implication for obesity. J Nutr 2000; 130 : 284S-8S.

6. Wing R, Matthews KA, Kuller LH, Meilahn EL, Plantinga PL. Weight gain at the time of menopause. Arch Intern Med I99 I; I5 I: 97 103.

7. Mahan LK, Arlin MT. Vitaminas. In: Mahan LK, Arlin MT, editores. Alimentos, nutrição e dietoterapia. $8^{\text {a }}$ ed. São Paulo: Roca; 1995.p. 99-143.

8. Heaney RP, Gallagher JC, Johnston CC, Neer R, Parfitt AM, Chir B, et al. Calcium nutrition and bone health in the elderly. Am J Clin Nutr 1982; 36: 986-1013.

9. Stevenson JC. Determinants of bone density in normal women: risk factors for future osteoporosis? Br Med J 1989; 298: 924-8.

10. Yates AA, Schlincker SA, Suitor CW. Dietary reference intakes: the new basisfor recommendation for calcium and related nutrients $B$ 
vitamins, and choline. J Am Diet Assoc 1998; 98:688-706.

II. World Health Organization (WHO). Report Meeting of the Subcommittees of the WPACHR on Environmental Health, Health Promotion, on Health Systems Research. Manilla, Philippines; 1996. Regional office for the Western Pacific. (Report Series n RS/95/ $\mathrm{GE} / 29$ (PHL).

12. Philippi ST, Szarfarc SC, Latterza AR. Virtual Nutri (software), versão I.0 for Windows. São Paulo. Departamento de Nutrição FSP/ USP; 1996.

13. Organización Mundial de la Salud (OMS). EI estado fisico: uso y interpretación de la antropometria. Ginebra; 1995. (Série de Informes Técnicos, n. 854).

14. National Research Council (NRC). Subcomittee on the tenth edition of the RDAs. Recommended dietary alowances 10th ed. Washington: National Academy Press; 1989.

15. Horwitz A. Guias alimentarias y metas nutricionales en el envejecimiento. Arch Latinoam Nutr 1988; 38: 723-49.

16. Guo SS, Zeller C, Chumlea WC, Siervogel RM. Aging, body composition, and lifestyle: the Fels Longitudinal Study. Am J Clin Nutr I 999; 70:405-II.

17. Stevens J. Impact of age on associations between weight and mortality. Nutr Rev 2000; 58: 129-37.

18. Stamler J. Epidemic obesity in the United States. Arch Intern Med 1993; 153:1040-4.

19. Mondini L, Monteiro CA. Mudanças no padrão de alimentação da população urbana brasileira (1962-1988). Rev Saúde Pública 1994; 28 : 433-39.

20. Kirk TR. Role of dietary carbohydrate and frequent eating in body-weight control. Proc Nutr Society 2000; 59: 349-58.

21. Monteiro CA, Mondini L, Souza ALM, Popkin BM. Da desnutrição para a obesidade: a transição nutricional no Brasil. In: Monteiro CA. Velhos e novos males da saúde no Brasil - A evolução do país e de suas doenças. São Paulo; Hucitec Nupens/USP; 1995. p. 247-55.

22. Poehlman ET, Michael JT, Gardner AW. Changes en energy balance and body composition at menopause: a controlled longitudinal study. Ann Intern Med 1995; 123: 673-5.

23. Williamson D. The 10-year incidence of overweight and major weight gain in U.S. adults. Arch Intern Med 1990; I 50:655-72.

24. AlbuJ, Shur M, Curi M, Murphy L, Heymsfield $S B$, Py-Sunyer FX. Resting metabolic rate in obese, premenopausal black women. Am J Clin Nutr 1997; 66:531-8.

25. Prentice AM, Jebb SA. Obesity in Britain: gluttony or sloth? BMJ 1 995;3 I I:437-9.

26. Lean MEJ. Pathophysiology of obesity. Proc Nutr Soc 2000; 59: 33I-6.

27. Organización Mundial de la Salud (OMS). Dieta, nutrición y prevención de enfermidades crónicas. Ginebra, 1990. (Série de Informes Técnicos, n. 797).

28. Johnston CC JR. Osteoporosis. In: Eskin BA, editor. The menopause: comprehensive management. 3th ed. New York: MacGraw Hill; 1994. p. 103-17.

29. Barzel US, Massey LK. Excess dietary protein can adversely affect bone. J Nutr 1998; 128:1051-3.

30. Nagant de Deuxchaisnes C. Nutritional factor in osteoporosis. In: Notelovitz M, Van Keep P, editors. The climateric in perspective. London: MTP Press Limited; 1986, p. 355-9.

3I. Szejnfeld VL. Osteoporose no climatério. In: Pinotti JA, Halbe HW, Hegg R, editores. Menopausa. São Paulo: Roca; 1995. p. I89-2II.

32. Melo NR. Propedêutica da mulher climatérica e pós-menopausal. In: Pinotti JA, Halbe HW, Hegg R, editores. Menopausa. São Paulo: Roca; 1995. p. 177-87.

33. Willett WC. Is dietary fat a major determinant of body fat. Am J Clin Nutr 1998;67: 556S- 62S.

34. McCrory MA, Fuss PJ, McCallum JE, Yao M, Vinken AG, Hays N, et al. Dietary variety within food groups: association with energy intake and body fatness in men and woman. Am J Clin Nutr 1999; 69: 440-7.

35. Flatt JP, Raverssin E, Acheson KJ, Jequier E. Effects of dietary fat on postprandial substrate oxidation and on carbohidrate and fat balances. J Clin Investig 1985; 76: I019-24.

36. Horton TJ, Drougas H, Brachey A, Reed GW, Peters JC, Hill JO. Fat and carbohidrate overfeeding in humans: different effects on energy storage. Am J Clin Nutr 1995; 62:19-29.

37. Bray G, Popkin BM. Dietary fat intake does affect obesity! Am J Clin Nutr 1998; 68: II57-73.

38. Rolls BJ, Hammer VA. Fat, carbohydrate and the regulation of energy intake. Am J Clin Nutr 1995; 62 (suppl. I): 1086S-95S.

39. Rolls BJ. The role of energy density in the overconsumption of fat. J Nutr 2000; 130 : 268S-7IS.

40. Drewnowski A, Greenwood MRC. Cream and sugar: human preference for high-fat foods. Physiol Behav 1983; 30: 629-33.

4I. Dawson-Hughes B, Harris SS. Calcium intake influences the association of protein take with rates of bone loss in elderly men and women. Am J Clin Nutr 2002; 75:773-9.

42. Heaney RP. Protein and calcium: antagonists or synergistics? Am J Clin Nutr 2002;75:609-I0.

43. Galeazzi MAM, Domene SMA, Sichiere R. Estudo multicêntrico sobre consumo alimentar. Revista do Núcleo de Estudos e Pesquisas em Alimentação/UNICAMP. Ministério da Saúde; 1997. (Volume Especial)

44. Felippe JR J. O excesso de ferro aumenta a incidência de doenças degenerativas da idade. Rev Med Biomol Radicais Livres 1996; 2: 6- 13.

45. HathcockJN. Vitamins and minerals: efficacy and safety. Am J Clin Nutr 1997; 66: 427-37.

Artigo recebido: 07/06/02

Aceito para publicação: 06/I I/02 